\title{
A PRELIMINARY REPORT ON TURBO PASCAL FOR THE MACINTOSH *
}

\author{
M. Ishaq Zahid \\ Department of Mathematics and Computer Science \\ The Citadel, Charleston, SC 29409
}

Borland International, Inc. released Turbo Pascal 1.0 for the Macintosh in 1986. Turbo Pascal for IBM PCs and compatibles has been in the market for awhile. The software usually sells for under $\$ 100.00$. Borland has a so-called Scholar Program through which a faculty member can purchase 10 or more copies for class use at a reduced price. I payed $\$ 39.95$ for my copy of the software under this program. The software comes on two disks and is accompanied by a manual. The first disk contains the Turbo compiler; the second has a set of utilities and a demo program. The manual has over 462 pages. Minimum system configuration is $256 \mathrm{~K}$ of memory and a single $400 \mathrm{~K}$ disk drive. The compilation of source code is fast. One of my programs of 11996 bytes and 526 lines split in two files compiled in 4.3 seconds. It took 11.5 seconds for the above program to compile and display the first response on the screen. Borland claims that Mac Turbo (Turbo Pascal for Macintosh computers) can compile 1420 lines of source code in 7.1 seconds. But, there is a catch: Turbo Pascal provides a switch to instruct the compiler to turn on or off the generation of range checking code. Unfortunately, the switch is OFF by default. This is an assault on Pascal. Here is an example of how Turbo Pascal can abuse a program. The primary reason of defining a subrange type, of say integer, is to keep variables of that type within bounds. If the variable $\mathrm{x}$ is declared of type $0 . .9$, then a logical error is committed if a value less than 0 or greater than 9 is assigned to $\mathrm{x}$ by the program. A run-time error should be produced in such a situation. This important feature of the language is particularly crucial for beginners. To burden the user with setting the switch for such range checking is a serious mistake. A slow running compiler is better than spending many hours devoted to debugging logical errors.

Another problem I faced was with the size of data area. The total area allocated for variables cannot exceed $32 \mathrm{~K}$ even if additional memory is available. This problem appears to have no solution, and is a serious drawback if you are thinking of writing programs that involve Mac Graphics or large data structures.

The software can be used to write "standard programs" as well as programs that exploit the bit-mapped graphics of the Macintosh. More serious applications can be written

\footnotetext{
${ }^{*}$ Received 7/20/87, revised 9/8/87, accepted 10/9/87.
} 
by using the event-driven methodology. To facilitate Mac-style programming, several libraries called units are provided on-line. Each unit has a specific purpose and consists of external and inline procedures and functions. Thus, for example, there is a QuickDraw unit which is a graphics package to draw lines, strings, rectangles,polygons, and regions. Another unit is ToolIntf which helps a user to deal with windows, menus, mouse control, and dialogue boxes. In order to use a unit in a program, the declaration part of the grammar includes the production

$$
\text { uses clause:: 'uses' identifier_list';' }
$$

which must appear right after the program heading. The user can also write his own units which then can be used in other programs.

The manual is divided into three parts as follows.

Part 1. User's guide (about 200 pages): This part describes the procedures that a beginner needs to start using Turbo Pascal (e.g., how to use the text editor, compiler and debugger). It also decribes how to write units and desk accessories for more complex applications.

Part 2. Reference Section (about 140 pages): This is a reference to the Turbo Pascal language.

Part 3. Appendices (6 of these on approximately 100 pages): Appendix A gives a comparison of Turbo Pascal with other Pascal implementations; Appendix B lists the error messages and codes; Appendix C explains compiler directives; Appendix D lists interface units; (each unit is essentially its source code without any explanations or details about the data structures and procedures involved); Appendix E lists the Macintosh character set; and Appendix F describes Turtle Graphics.

There is a problem when a program consists of more than one file. A program file can be divided into several smaller files. One of these files, called the main file, contains the overall structure of the program. The other files, called include files, are the various pieces of the program. To compile and run such a program, the main file must be open. Once a program is compiled, its object code resides in the memory. This object code is used in any subsequent runs of the program. The run option of the Mac Turbo actually consists of two commands, compile and execute. The run option works as follows.

if the current program file is the same from the last run

then execute

else compile and execute.

The problem is with modifications of include files. If the main file is the same from the last run but an include file is modified, the run command executes the program without 
compilation. To avoid this problem I ended up deleting and adding a character in the main file before running it.

The run-time environment poses another problem. Programmers and novices alike unintentionally write infinite loops in their programs. Turbo Pascal does not provide a graceful exit for such run-away programs. You must guard against such runtime errors when programming or shut the machine off and start over when infinite loops begin. Of course, when you start over, you may not have the latest versions of your files on your disks.

Borland could have done a better job on the manual. All of Part 1 and in fact the rest as well delves on one single program for all of the illustrations. Almost all illustrations come from the program demo on the utilities disk. The documentation needs a variety of programs to illustrate different features. All programs are not going to be written with all the features available on the system. The source code of the demo program itself is of little educational value. The demo program and the relevant illustrations of the manual are typical of vendors who inconvenience consumers by releasing their products prematurely and eventually obtain additional sales with subsequent releases. I recently received a letter from Borland about their "new" software, Turbo Tutor for the Macintosh, which would teach Turbo Pascal. The advertisement begins with: "Learn to program in Pascal from the people who invented Turbo Pascal for the Macintosh!"

Preliminary Conclusions:

1. If you want to do simple Pascal programming with graphics, Turbo Pascal for the Macintosh is an excellent choice. It is easy to learn and program on the Macintosh. Despite its pitfalls, Turbo Pascal is one of the better alternatives for introductory computer science classes.

2. If you want to write serious applications in Pascal which utilize mouse control, windows, menus, etc., then use Turbo Pascal, but beware; you either have to be a Macintosh expert or have access to references on the internals of the Macintosh such as Inside Macintosh, Vol 1- 4, Addison-Wesley, 1986. 


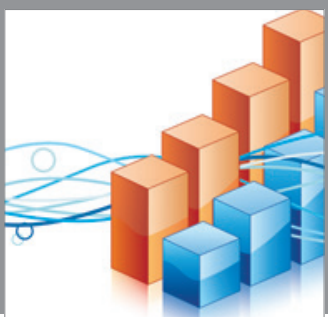

Advances in

Operations Research

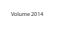

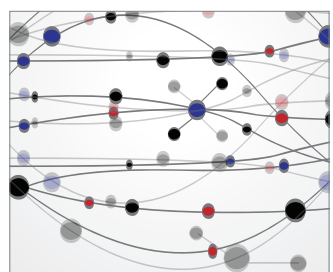

\section{The Scientific} World Journal
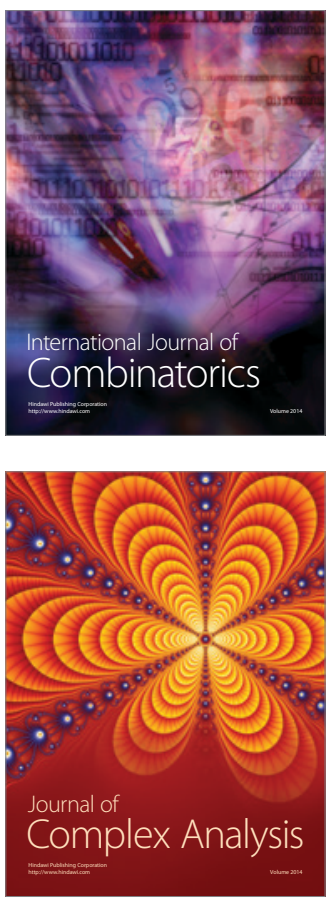

International Journal of

Mathematics and

Mathematical

Sciences
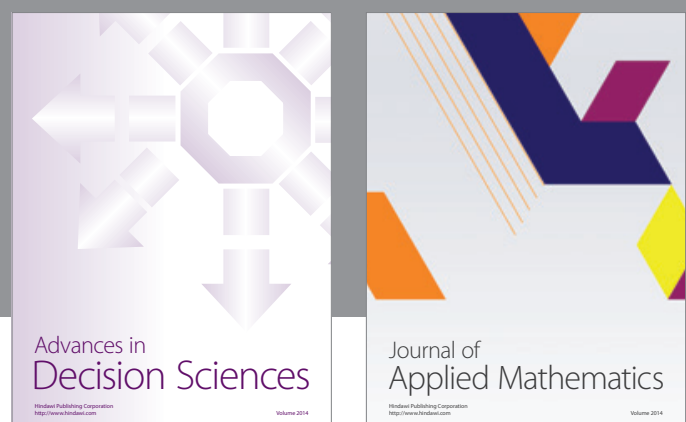

Journal of

Applied Mathematics
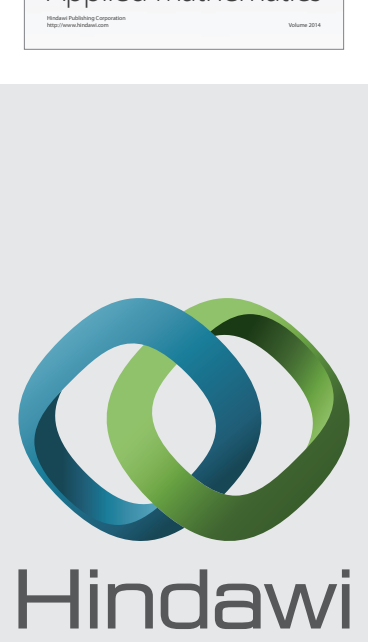

Submit your manuscripts at http://www.hindawi.com
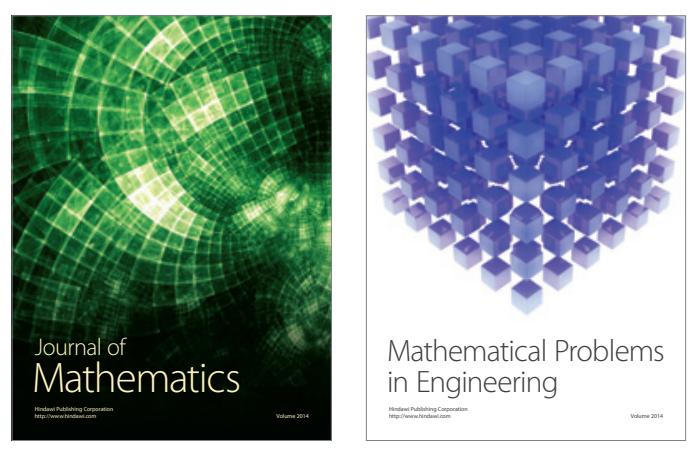

Mathematical Problems in Engineering
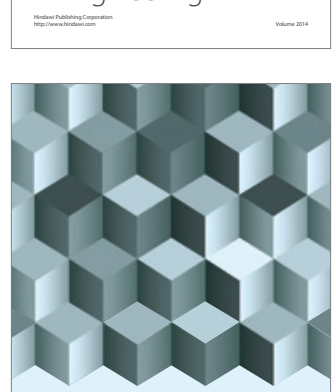

Journal of

Function Spaces
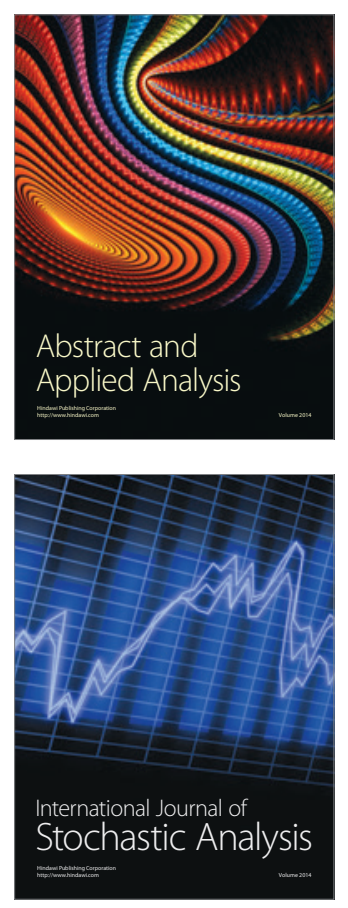

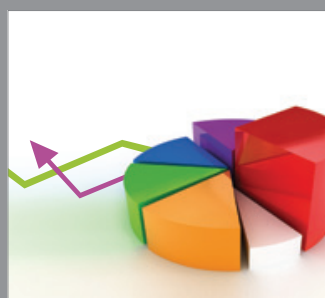

ournal of

Probability and Statistics

Promensencen
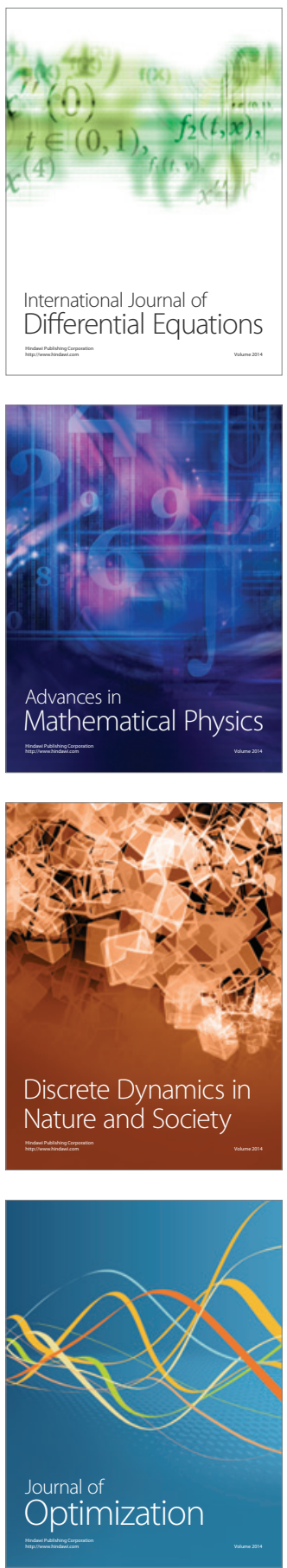